\title{
Diagnosis of Tubercular Lymphadenopathy by Fine Needle Aspiration Cytology, Acid-Fast Staining and Mantoux Test
}

\author{
Lakhey $\mathrm{M}^{1}$ 'Bhatta CP,' Mishra S' \\ 'Department of Pathology, Medicare National Hospital and Research Centre, Kathmandu, Nepal.
}

\section{ABSTRACT}

Introduction: This study was undertaken to evaluate the role of Ziehl-Neelsen stain (for acid-fast bacilli), and Mantoux test in diagnosing tubercular lymphadenopathy on FNAC.

Methods: FNAC was performed on patient with superficial lymphadenopathy. Ziehl-Neelsen stain for acid fast bacilli was done in all cases where cheesy or purulent material was aspirated and smears showed granulomatous lymphadenitis. A Mantoux test was also done in these patients. A Mantoux test was further done in patients of reactive lymphadenopathy where the lymph node size was more than $1 \mathrm{~cm}$. If the result was positive, a repeat FNAC was performed. When the repeat FNAC showed ill-defined granulomas, excision biopsy was done.

Results: Tubercular lymphadenopathy was seen in 122 (48.2\%) cases. Acid- fast bacilli were found in $71(58.1 \%)$ cases. Mantoux test was positive in $112(91.8 \%)$ cases. A repeat FNAC was done in seven of 11 patients where the cytologic features of tuberculosis were not seen but the lymph node size was more than $1 \mathrm{~cm}$ and Mantoux test was positive. The repeat FNAC showed ill-defined granulomas without necrosis. Excision biopsy done in these patients diagnosed them as tubercular lymphadenitis in five of the seven cases, the remaining two cases being diagnosed as reactive lymphadenitis.

Conclusions: FNAC coupled with Ziehl - Neelsen staining for AFB and Mantoux test improves the diagnostic efficiency for tubercular lymphadenopathy.

Key Words: FNAC, lymphadenopathy, mantoux test, tuberculosis, Ziehl-Neelsen stain

\section{INTRODUCTION}

Tuberculosis is a major public health problem in Nepal. Tubercular lymphadenitis is a common manifestation of extrapulmonary tuberculosis and one of the main causes of lymphadenopathy.

The utility of Fine Needle Aspiration Cytology (FNAC) in the diagnosis of tubercular lymphadenitis is being described increasingly by many authors. ${ }^{1-5}$ It is a simple out patient diagnostic procedure which is well accepted by patients and has practically no complications. ${ }^{6}$

Performing Ziehl-Neelsen (Z-N) staining for acid-fast bacilli (AFB) in suspected cases of tuberculosis is of particular importance in view of the high prevalence of tuberculosis in our country, atypical presentation of tuberculosis and the fact that AFB is seen in most cases
Correspondence:

Dr. Mamta Lakhey

Department of Pathology

Medicare National Hospital and Research Centre

Chabahil, Kathmandu, Nepal.

Email: mlakhey65@yahoo.com

Phone: $977-9851005854$ 
Lakhey et al. Diagnosis of Tubercular Lymphadenopathy by Fine Needle Aspiration ...

of purulent aspirates where granulomas or caseous necrosis are not seen. Mantoux test has significance in the diagnosis of tuberculosis, especially where the cytomorphologic features of tuberculosis are illdefined.

The aim of this study was to evaluate the role of FNAC in diagnosing tuberculous lymphadenopathy and to determine the utility of Z-N stain and Mantoux test in suspicious cases.

\section{METHODS}

A prospective observational study was conducted in the Department of Pathology, in Kathmandu Medical College from April 2005 to March 2008. Ethical approval and consent was taken. FNAC was performed on 286 patients who presented with superficial palpable lymph nodes with informed consent of the patient. Aspiration was done using a 20-23 gauge needle and disposable $10 \mathrm{ml}$ plastic syringe. In all cases, alcohol fixed and air-dried smears were made: alcohol fixed slides were stained by Papanicolaou (PAP) method and air-dried smears were stained by May Grunwald- Giemsa (MGG). Special stain for acid fast bacilli (Z-N stain) was done in all cases where purulent or cheesy material was aspirated and smears showed granulomatous lymphadenitis. A Mantoux test was also done in these patients. All the stained smears were evaluated by a pathologist and a diagnosis given based on cytomorpological features. In addition, a Mantoux test was done in cases of nonspecific lymphadenopathy where the lymph node size was more than $1 \mathrm{~cm}$. If the result of the Mantoux test was positive (more than $10 \mathrm{~mm}$ ), a repeat FNAC was done to rule out tubercular etiology. When the repeat FNAC showed ill-defined granulomas without necrosis, excision biopsy was done in these patients.

\section{RESULTS}

Out of the 286 cases, 253 (88.4\%) were nonneoplastic lymphadenopathy. Among these cases, 126 $(49.8 \%)$ were of reactive nature and $122(48.2 \%)$ were tubercular. Thirty three $(11.6 \%)$ cases were malignant lymphadenopathy: 10 (30.3\%) were lymphomas and 23 $(69.7 \%)$ were metastasis to lymph nodes (Table 1$)$

The age of the patients with tubercular lymphadenitis ranged from three to 79 years, the majority of the cases being in the third and fourth decade of life $(63.1 \%)$. Out of the 122 cases of tuberculosis, 71 were reported in females and 51 were reported in males, with a female to male ratio of $1.4: 1$. The cervical group of lymph node was most commonly involved in the present study (92\%). $95 \%$ of cases of reactive lymphadenopathy showed discrete lymph nodes as compared to tubercular lymphadenopathy which showed matted lymph nodes in $63 \%$ cases.
Table 1. Cytological diagnosis of 286 cases of lymphadenopathy

\begin{tabular}{lcc}
\hline Cytological diagnosis & $\begin{array}{c}\text { Number of } \\
\text { cases }\end{array}$ & $\begin{array}{c}\text { Percentage } \\
\text { (\%) }\end{array}$ \\
\hline Reactive hyperplasia & 126 & 44 \\
Tuberculous Iymphadenitis & 122 & 42.6 \\
Non-Hodgkin's Lymphoma & 8 & 2.7 \\
Hodgkin's Lymphoma & 2 & 0.6 \\
Metastatic carcinoma & 23 & 8.0 \\
Suppurative Iymphadenitis & 3 & 1.0 \\
Rosai-Dorfman's Disease & 1 & 0.3 \\
Kikuchi's lymphadenitis & 1 & 0.3 \\
\hline
\end{tabular}

The aspirates from lymph nodes were diagnosed as tubercular lymphadenopathy based on the presence of epithelioid cell granulomas with or without Langhan's giant cells, with necrosis against a background of lymphoid cells or epitheloid cell granulomas without necrosis and caseation or only necrotic material consisting of diffuse granular debris. The cytological findings of 122 cases of tubercular lymphadenitis are shown in Table 2.

Out of the 122 cases of tubercular lymphadenitis, AFB was found in 71 cases (58.1\%). AFB on Z-N stain was seen as short and long beaded rods. The AFB positivity rate was higher $(82.3 \%)$ when cheesy, necrotic or puslike material was aspirated.

Of the 122 cases of tuberculosis, 112 (91.8\%) were Mantoux positive (Mantoux positive was considered when erythema was present along with induration which was more than or equal to $10 \mathrm{~mm}$ in diameter, 72 hours after intradermal injection of $0.1 \mathrm{~mL}$ of purified protein derivative (PPD) on the flexor surface of forearm).

The lymph node size was less than $1 \mathrm{~cm}$ in $82 \%$ of reactive lymphadenopathy where as it was over $1 \mathrm{~cm}$ in $90 \%$ of tubercular lymphadenopathy. A repeat FNAC was done in seven of 11 patients where the cytologic features of tuberculosis were not seen but the lymph node size was more than $1 \mathrm{~cm}$ and Mantoux test was positive. The repeat FNAC showed ill-defined granulomas without necrosis. Excision biopsy done in these patients diagnosed them as tubercular lymphadenitis in five of the seven cases, the remaining two cases being diagnosed as reactive lymphadenitis. Four patients did not come for a repeat FNAC as advised.

\section{DISCUSSION}

Tubercular lymphadenopathy remains the commonest extrapulmonary manifestation of tuberculosis in children and adults. Although histopathology remains the gold 
Lakhey et al. Diagnosis of Tubercular Lymphadenopathy by Fine Needle Aspiration ...

Table 2. Cytological findings of 122 cases of tubercular lymphadenitis

\begin{tabular}{llllc}
\hline Cytological findings & Number of cases & Percentage (\%) & \multicolumn{2}{c}{ Z-N positive } \\
& & & No. & \% \\
\hline Granulomas alone & 31 & 25.5 & 05 & 16.1 \\
Granulomas and necrosis & 57 & 46.7 & 38 & 66.6 \\
Necrosis alone & 34 & 27.8 & 28 & 82.3 \\
Total & 122 & 100 & 71 & - \\
\hline
\end{tabular}

standard for diagnosis of cervical lymphadenopathy, its feasibility is limited as it is time consuming and an invasive procedure. Now it has been replaced by FNAC which is a simple, safe, minimally invasive and rapid diagnostic technique.

In our study, tubercular lymphadenopathy with 122 $(48.2 \%)$ cases was the second most common cause of peripheral lymphadenopathy, reactive lymphadenopathy with $126(49.8 \%)$ cases being the commonest. Tubercular lymphadenitis is one of the most common types of lymphadenopathy in developing countries, ${ }^{7-10}$ in sharp contrast with a very low frequency of $1.6 \%$ in Western studies. ${ }^{11}$ The high rate of tubercular lymphadenitis in our country is due to low socioeconomic status, illiteracy, incomplete treatment, resistance and increased incidence of HIV. In our study, it was seen most frequently in the third and fourth decade of life $(63.1 \%)$ with a female / male ratio of (1.4:1). The cervical lymph node was the most common site involved $(92 \%)$ followed by axilla $(5 \%)$ which is similar to observations by other workers. ${ }^{12}$

The cytological diagnosis of tubercular lymphadenitis is usually based on the presence of epithelioid cell granulomas with or without Langhan's giant cells and with or without necrosis. Even in the absence of epithelioid cells, necrotic material has proved to be useful as it yields the highest yield of AFB positivity. ${ }^{13}$ In our study, AFB staining in cases of tuberculosis showed a positivity rate of $58.1 \%$. Highest yield of AFB was seen in cases which showed necrosis and purulent or pus-like aspirate $(65.8 \%)$, as reported earlier. ${ }^{13-5}$ Many of these cases where granulomas were not present or occasionally seen could have been misdiagnosed as suppurative lymphadenitis in the absence of Z-N stain. Acid fast bacilli were usually seen in areas of necrosis and degeneration away from the epithelioid cells. The bacilli were identified as short, stumpy or long beaded rods. These findings correlated with the observation by Rajshekaran et al. ${ }^{15}$ In our study, the maximum load of AFB $(+++)$ was seen in immune-compromised patients which included seven patients of HIV and five patients on immunosuppressive therapy following kidney transplant for chronic kidney disease (CKD). The smears of these patients revealed mainly necrosis with occasional ill-defined granulomas.
Mantoux test in our study was found to be positive in $112(91.8 \%)$ cases. The utility of the Tuberculin test as a diagnostic criterion has been debated by many authors. Bedi et $\mathrm{al}^{16}$ have stated limited role of tuberculin test in diagnosis of tubercular lymphadenopathy. In their study, 20 confirmed tuberculous cases gave a tuberculin reaction of $0-9 \mathrm{~mm}$, which is usually reported as negative, i.e. shows uninfected status. Ahmed et $\mathrm{al}^{17}$ found Mantoux positivity in only $54.9 \%$ of tubercular lymphadenopathy. They attributed this to the fact that in majority of patients with tuberculosis, the cellular immune response could be depressed. This however meant that a negative Mantoux test cannot be relied upon to exclude tuberculosis. Bedi et $\mathrm{al}^{16}$ and Park $^{18}$ are also supportive of this view on Mantoux test in tuberculosis. Garde et al $^{19}$ have highlighted the significance of Mantoux test in the diagnosis of tuberculosis in children: 10 out of 15 cases were Mantoux positive. In our study a high percentage of Mantoux positivity (91.8\%) was seen in patients of tubercular lymphadenopathy, many of which showed strong positivity (more than $20 \mathrm{~mm}$ ). Cases of "reactive lymphadenopathy" with lymph nodes measuring more than $1 \mathrm{~cm}$ in size and featuring a positive Mantoux test proved to be tuberculosis on biopsy highlighting the utility of Mantoux test especially in suspicious cases. Mantoux positive $(28 \mathrm{~mm})$ was seen in one case of salivary gland carcinoma with lymph node metastasis. Three percent of the cases diagnosed as reactive lymphadenopathy also showed Mantoux positivity. Due to the real possibility of a false-positive Mantoux skin test, these patients were followed up with a chest $x$-ray and sputum test which failed to diagnose concurrent tuberculosis. Thus, although being a simple and valuable tool for detecting tuberculosis exposure and infection, testing positive does not always indicate tuberculosis. There are several factors that can produce a falsepositive TB skin test reaction some being infection with non-tuberculous mycobacteria and past inoculation with BCG vaccine.

\section{CONCLUSIONS}

The present study suggests that FNAC coupled with Z$\mathrm{N}$ staining for AFB is an excellent method for diagnosing tubercular lymphadenitis. Mantoux test, although 
Lakhey et al. Diagnosis of Tubercular Lymphadenopathy by Fine Needle Aspiration ...

controversial, is of significance and utility especially in doubtful cases. Other ancillary investigations like mycobacterial culture, lymph node biopsy and polymerase chain reaction can be reserved for cases, in which there is strong clinical suspicion with equivocal result of FNAC and acid-fast stain. FNAC should be coupled with Z-N stain in all cases of granulomatous lymphadenitis and in aspirates yielding necrotic and purulent material before making a cytological diagnosis. Mantoux test should be done on patients with lymphadenopathy of more than
$1 \mathrm{~cm}$ showing features of non-specific lymphadenitis. If the result is positive, a repeat FNAC/biopsy should be done before a final diagnosis.

\section{ACKNOWLEDGEMENTS}

The authors gratefully acknowledge the help of Parima Dangal and the laboratory technical staff for their contribution in this study.

\section{REFERENCES}

1. Bailey TM, Akhtar M, Ali M. Fine needle aspiration biopsy in the diagnosis of tuberculosis. Acta Cytol. 1985;29(5):732-6.

2. Finfer M, Perchick A, Burstein DE. Fine needle aspiration biopsy diagnosis of tuberculous lymphadenitis with and without the acquired immune deficiency syndrome. Acta Cytol. 1991;35:325-32.

3. Metre MS, Jayaram G. Acid fast bacilli in aspiration smears from tuberculous lymph nodes: An analysis. Acta Cytol. $1987 ; 31: 17-9$

4. Shariff S, Thomas JA. Fine needle aspiration cytodiagnosis of clinically suspected tuberculosis. Acta Cytol. 1991;35:333-6.

5. Singh JP, Chaturvedi NK, Das A. Role of fine needle aspiration cytology in the diagnosis of tuberculous lymphadenopathy. Ind J Pathol Microbiol. 1989;32:100-4.

6. Harding Rains AJ, David Ritchie H, editors. Bailey and Love's Short Practice of Surgery. 19th ed. United Kingdom: Hodder Arnold;1985. p. 601-10.

7. Prasad RR, Narasimhan R, Sankran V, Veliath AJ. Fine needle aspiration cytology in the diagnosis of superficial lymphadenopathy: an analysis of 2418 cases. Diagno Cytol. 1993;15:382-6.

8. Paul PC, Goswami BK, Chakrabarty S, Giri A, Pramanik R. Fine needle aspiration cytology of lymph nodes: An institutional study of 1448 cases over a five year period. J Cytology. 2004;21:187-90.

9. Gupta AK, Nayar M, Chandra M. Critical appraisal of Fine needle aspiration cytology in tuberculosis. Acta Cytol. 1992;36:391-4.
10. Bhaskran CS, Kumar GH, Sreenivas M, Rajni K, Rao G, Aruna CA. Fine needle aspiration cytology review of 1731 cases. Ind J Pathol Microbial. 1990;33:387 - 97.

11. Kline TS, Khannan V, Line IK. Lymphadenopathy and aspiration biopsy cytology review of 376 superficial lymph nodes. Cancer. 1984;54:1076-81.

12. Sheikh MM, Ansari Z, Ahmed P, Tyagi SP. Tuberculous lymphadenopathy in Children. Indian Pediatr. 1981;18:293-7.

13. Metre MS, Jayram G. Acid fast bacilli in aspiration smears from tuberculous lymphnodes. Acta Cytologica. 1987;31:17-9.

14. Prasoon D. Acid fast bacilli in FNA smears from tuberculous lymphnodes: where to look for them. Acta Cytologica. 2000;44:297-300.

15. Rajsekaran S, Gunasekaran M, Bhanumati V. Tuberculous cervical lymphadenitis in HIV positive and negative patients. Ind J Tub. 2001;48:201-4.

16. Bedi RS, Thind GS, Arora VKA. Cilnico- pathological study of superficial lymphadenopathy in Northern India. Ind J Tub. 1987;34:189-91.

17 Ahmad SS, Akhtar S, Akhtar K, Naseen N, Mansoor T. Study of fine needle aspiration cytology in lymphadeopathy with special reference to Acid-fast staining in cases of Tuberculosis. JK Science. 2006;8:157-9.

18. Park K. Textbook of preventive and social medicine. 17th ed. Jabalpur(India):M/S Banarasi Das Bhanot; 2002. p. 202-6.

19. Gadre DV, Singh UR, Saxena K, Bhatia A, Talwar V. Diagnosis of tubercular cervical lymphadenopathy by FNAC, microscopy and culture. Ind J Tub. 1991;38:25-7. 